\title{
CATARATAS PEDIÁTRICAS: ESTUDIO EPIDEMIOLÓGICO Y DIAGNÓSTICO. ANÁLISIS RETROSPECTIVO DE 79 CASOS
}

\author{
PEDIATRIC CATARACTS: EPIDEMIOLOGY AND DIAGNOSIS. \\ RETROSPECTIVE REVIEW OF 79 CASES
}

\author{
PERUCHO-MARTÍNEZ S ${ }^{1}$, DE-LA-CRUZ-BERTOLO J ${ }^{2}$, TEJADA-PALACIOS P ${ }^{2}$
}

\begin{abstract}
RESUMEN
Objetivo: Determinar las características epidemiológicas, diagnósticas y terapéuticas de un grupo de cataratas de nuestro medio con el propósito de mejorar el pronóstico visual de estos pacientes.

Métodos: Estudio retrospectivo de 79 casos de cataratas pediátricas durante un periodo de 18 años (1986-2004). Hemos diferenciado dos grupos etiológicos de trabajo: cataratas congénitas y cataratas adquiridas.

Resultados: La causa más frecuente entre las congénitas fue la idiopática $(68 \%)$ y la más frecuente de las adquiridas fue traumática (90\%). La morfología más frecuente de las congénitas fue la nuclear $0,31(31 \%)$. El $56 \%$ de las cataratas congénitas fueron bilaterales. El 27\% de las cataratas congénitas se asociaban a otras anomalías oculares y la más frecuente fue el microftalmos. El signo clínico de presentación más frecuente de las cataratas congénitas fue la leucocoria, en 0,44 (44\%). El 75\% $(0,75)$ de las cataratas congénitas de nuestro medio tardan menos de un mes en diagnosticarse desde la manifestación clínica. El 58\% $(0,58)$ de las cataratas congénitas se trataron con cirugía y el $50 \%$ de
\end{abstract}

\begin{abstract}
Purpose: To determine the epidemiology, diagnosis and treatment features in a group of pediatric patients with cataracts treated at our hospital. The aim was to improve the visual prognosis in these patients.

Methods: 79 children with cataracts were reviewed retrospectively during an 18 year period (19862004). This involved patients with congenital cataracts and those who acquired them later. Most of the latter group had a traumatic etiology (90\%).

Results: The etiology of most cataracts was idiopathic $(68 \%)$ for the congenital group and traumatic $(90 \%)$ for the acquired group. Congenital cataracts were frequently nuclear in type $(31 \%)$ with $56 \%$ being bilateral. $27 \%$ of the congenital group were associated with dysmorphic eye features, the most frequent being microphthalmos. The most frequent presenting feature was leucokoria, seen in $44 \%$ of patients. $75 \%$ of congenital cataracts were diagnosed within one month of clinical manifestation. 58\% of the congenital cataracts were treated by surgery and $50 \%$ of these were performed within one month of the diagnosis. $90 \%$ of the acquired
\end{abstract}

Recibido: 27/2/06. Aceptado: 17/1/07

Servicio de Oftalmología. Hospital Universitario 12 de Octubre. Madrid. España.

1 Licenciado en Medicina.

2 Doctor en Medicina.

Comunicación presentada parcialmente en el 17 Congreso de la Sociedad Española de Estrabología (Madrid 2004).

Correspondencia:

Susana Perucho Martínez

C/. Cabo San Vicente, $10,2 .{ }^{\circ} \mathrm{C}$ derecha

28924 Alcorcón (Madrid)

España

E-mail: sperucho@ya.com 
ellas tardaron menos de 1 mes en operarse. El 90\% $(0,9)$ de las adquiridas se trataron con cirugía y el 95\% de ellas tardaron menos de un mes en operarse. Conclusiones: En nuestra serie conseguimos un diagnóstico y tratamiento precoz en un alto porcentaje de pacientes. El pronóstico visual de estos niños viene determinado por la precocidad en el diagnóstico y el tratamiento, es por ello que debemos continuar intentando acortar este período de tiempo y conseguir que todos los niños sean diagnosticados y tratados precozmente.

Palabras clave: Catarata, pediátrica, congénita, lente intraocular, pseudofaquia, extracción de catarata. cataracts were treated surgically, and $95 \%$ of these were performed less than one month after diagnosis. Conclusions: We attained a prompt diagnosis and treatment in a high percentage of cases. Prompt diagnosis and treatment will determine the visual prognosis of these patients. We must continue trying to shorten this period of time in order that all children with this condition are diagnosed and treated urgently and efficiently (Arch Soc Esp Oftalmol 2007; 82: 37-42).

Key words: Cataract, pediatric, congenital, intraocular lens, pseudophakia, cataract extraction.

\section{INTRODUCCIÓN}

Las catataras congénitas de desarrollo temprano así como las cataratas traumáticas son enfermedades oculares frecuentes (1,4-6,3 por cada 100.000 nacidos vivos según la población de estudio) y constituyen una causa importante de disminución de agudeza visual (AV) en la infancia (1). La prevalencia de ceguera (agudeza visual con corrección menor a 0,05 ) por catarata infantil puede estar alrededor de 1 a 4/10.000 niños en los países subdesarrollados y entre 0,1 a $0,4 / 10.000$ niños en los países industrializados (2-5).

Tienen unas características especiales y su manejo es completamente distinto a las del adulto.

La principal causa de pérdida de visión relacionada con cataratas congénitas o infantiles es la ambliopía. Los primeros meses de la vida representan un período crítico en el desarrollo visual, puesto que las áreas visuales del encéfalo experimentan maduración rápida en respuesta a las señales visuales procedentes de los ojos $(6,7)$. La borrosidad de la imagen retiniana en uno o ambos ojos durante este período crítico originará una ambliopía irreversible.

$\mathrm{Su}$ diagnóstico precoz es una prioridad internacional (8), pues de ello depende directamente el pronóstico visual de estos pacientes. Esta prioridad ha llevado a elaborar estrategias de prevención e identificación tanto en países desarrollados como en países en vías de desarrollo (9).

Se han analizado 79 casos de cataratas pediátricas de la consulta de oftalmología pediátrica de nuestro área hospitalaria, determinando su incidencia en nuestro área, su etiología y morfología, características del diagnóstico y tratamiento con el propósito de mejorar el pronóstico visual de estos pacientes.

\section{SUJETOS, MATERIAL Y MÉTODOS}

Estudio retrospectivo de 79 casos de cataratas pediátricas presentes en nuestro medio durante un periodo de diagnóstico y seguimiento de 18 años (1986-2004).

Se han definido dos grupos etiológicos de trabajo:

Grupo de cataratas congénitas, dentro de las cuales estarían incluidas las idiopáticas, genéticas, asociadas a síndromes y las debidas a infecciones intrauterinas.

Grupo de cataratas adquiridas, dentro de las cuales estarían incluidas las cataratas traumáticas y metabólicas.

En cada uno de los grupos etiológicos se han analizado los siguientes parámetros: sexo, antecedentes familiares, localización, morfología y características de las cataratas, asociación a otras malformaciones oculares, forma de presentación, edad de presentación clínica, edad del diagnóstico por parte del pediatra u oftalmólogo, tratamiento quirúrgico o conservador y edad en la que se realiza la cirugía.

Se ha empleado el término de leucocoria como signo de presentación clínica y denominamos leucocoria en este trabajo, a cualquier opacidad cristaliniana, no al típico reflejo blanquecino (leucocoria) que producen otro tipo de patologías oftalmológicas pediátricas como el retinoblastoma, PVPH, toxoplasmosis,... 
Se ha realizado un análisis estadístico descriptivo en el que se presentan distribuciones de frecuencias absolutas y relativas. Se ha empleado el test de Chicuadrado para contrastar la significación estadística de las diferentes proporciones. El programa informático de análisis estadístico empleado es el SAS v 8.02; SAS Institute Inc., (Cary, NC, USA).

\section{RESULTADOS}

En el análisis de grupos etiológicos, 59/79 $(74,36 \%)$ fueron cataratas congénitas (idiopáticas, hereditarias, asociadas a síndromes y asociadas a infecciones intrauterinas) y 20/79 $(25,64 \%)$ fueron adquiridas (traumáticas y metabólicas)

En las cataratas congénitas encontramos que 0,68 (68\%) son idiopáticas, 0,17 (17\%) hereditarias, 0,03 (3\%) asociadas a infecciones intrauterinas (dos casos de rubéola congénita) y 0,12 (12\%) asociadas a síndromes (nueve casos asociados a Síndrome de Down y un caso asociado a Síndrome de Rubisntein-Taybi).

En las adquiridas 0,9 (90\%) son de causa traumática y $0,1(10 \%)$ son de causa metabólica (tres casos con enfermedades reumatoides y renales tratadas con altas dosis de corticoides).

Con respecto a los antecedentes familiares, 0,27 $(27,11 \%)$ cataratas congénitas si los tenían mientras que $0,73(72,8 \%)$ no los tenían.

En cuanto a la morfología de las cataratas congénitas, $0,31(31 \%)$ fueron cataratas nucleares, 0,15 (15\%) fueron polares, $0,08(8 \%)$ fueron subcapsulares; el resto de morfologías, menos frecuentes, quedan recogidas en la tabla I. En el grupo de las adquiridas, 0,05 casos $(5 \%)$ era de tipo nuclear, 0,05 casos $(5 \%)$ era de tipo polar, 0,35 casos $(35 \%)$ eran de tipo subcapsular, $0,35(35 \%)$ casos tenían otras formas y 0,2 casos (20\%) desconocíamos su morfología.

Tabla I. Frecuencia relativa de las cataratas congénitas según su morfología

\begin{tabular}{lcr}
\hline Morfología & Número & Porcentaje \\
\hline Nuclear & 18 & $30,50 \%$ \\
Polar & 9 & $15,25 \%$ \\
Zonular & 1 & $1,69 \%$ \\
Subcapsular & 5 & $8,47 \%$ \\
Completa & 2 & $3,38 \%$ \\
Otras & 7 & $11,86 \%$ \\
Desconocida & 17 & $28,81 \%$ \\
Total & 59 & $100,00 \%$ \\
\hline \hline
\end{tabular}

Con respecto a la localización encontramos que en el grupo de las cataratas congénitas 0,56 (56\%) eran bilaterales y $0,4(44 \%)$ eran unilaterales. Sin embargo en el grupo de las adquiridas 0,85 (85\%) eran unilaterales y tan solo $0,15(15 \%)$ eran bilaterales. No se encontraron resultados estadísticamente significativos en cuanto a la aparición en ojo derecho (OD) u ojo izquierdo $(\mathrm{OI})$ en los casos de cataratas unilaterales. En el grupo de las congénitas 0,2 (20\%) eran en ojo derecho y 0,24 (24\%) eran en ojo izquierdo. En el grupo de las adquiridas 0,5 (50\%) eran en ojo derecho y 0,35 (35\%) eran en ojo izquierdo.

Se analizó la asociación de las cataratas congénitas a otras anomalías oculares encontrando que 0,27 (27\%) presentaban otras anomalías asociadas. La anomalía más frecuentemente asociada era el microftalmos que aparecía en 0,44 casos (44\%). Otros tipos de anomalías oculares asociadas menos frecuentes fueron persistencia de membrana pupilar que aparecía en 0,31 (31\%) casos, glaucoma congénito en 0,2 casos (19\%), persistencia de vítreo primario hiperplásico en 0,06 casos (6\%).

Con respecto a la forma de presentación clínica de las cataratas en nuestro medio se observó que la más frecuente entre las congénitas es la leucocoria (44\%) y entre las adquiridas es el antecedente de traumatismo previo (tabla II).

Se identificó a qué edad se presentaba el primer signo clínico obteniendo los siguientes resultados, en el grupo de las congénitas $17 \%$ de los casos se presentaron al nacimiento, $40 \%$ durante los primeros 3 meses de vida, $13,5 \%$ de 3-6 meses, 5\% entre 6-12 meses, $11,8 \%$ entre $12-48$ meses y $5 \%$ con más de 48 meses. En el grupo de las adquiridas ningún caso apareció al nacimiento, sólo un caso (5\%) apareció durante el primer año de vida, 30\% de los casos entre 12-48 meses de vida, 20\% entre 48-96 meses y $45 \%$ de los casos aparecieron con más de 96 meses de vida.

Conociendo la edad de la presentación clínica y la edad en la que se diagnostican, es posible saber cuánto tiempo se ha tardado en diagnosticar la patología en nuestro medio, desde que se presentó clínicamente. De esta manera pudimos ver que en el grupo de las cataratas congénitas, en 0,67 (70\%) de los casos el diagnóstico se hizo como máximo en las 2-3 primeras semanas tras la aparición clínica, en $0,06(7 \%)$ pasó un mes, en 0,06 (7\%) pasaron 13 meses, 0,01 (2\%) pasaron 3-6 meses y en 0,13 (14\%) pasaron más de 6 meses entre la aparición clínica y el diagnóstico. 
Tabla II. Forma de presentación de las cataratas infantiles según su diagnóstico etiológico: cataratas congénitas y adquiridas

\begin{tabular}{|c|c|c|c|c|}
\hline \multirow[t]{2}{*}{ Forma presentación } & & \multicolumn{2}{|c|}{ Grupos etiologicos } & \multirow[t]{2}{*}{ Total } \\
\hline & & Congénitas & Adquiridas & \\
\hline \multirow[t]{2}{*}{ Leucocoria } & Número & 26 & 2 & 28 \\
\hline & Porcentaje & $44,00 \%$ & $10,00 \%$ & $35,44 \%$ \\
\hline \multirow{2}{*}{ Nistagmus } & Número & 7 & 0 & 7 \\
\hline & Porcentaje & $11,80 \%$ & $0,00 \%$ & $8,86 \%$ \\
\hline \multirow[t]{2}{*}{ Estrabismo } & Número & 9 & 0 & 9 \\
\hline & Porcentaje & $15,25 \%$ & $0,00 \%$ & $11,39 \%$ \\
\hline \multirow[t]{2}{*}{ Disminución AV } & Número & 2 & 2 & 4 \\
\hline & Porcentaje & $3,38 \%$ & $10,00 \%$ & $5,06 \%$ \\
\hline \multirow[t]{2}{*}{ No signo } & Número & 7 & 16 & 23 \\
\hline & Porcentaje & $11,86 \%$ & $80,00 \%$ & $29,11 \%$ \\
\hline \multirow[t]{2}{*}{ Desconocido } & Número & 8 & 0 & 8 \\
\hline & Porcentaje & $13,50 \%$ & $0,00 \%$ & $10,12 \%$ \\
\hline \multirow[t]{2}{*}{ Total } & Número & 59 & 20 & 79 \\
\hline & Porcentaje & $100,00 \%$ & $100,00 \%$ & $100,00 \%$ \\
\hline
\end{tabular}

En las cataratas adquiridas se observó que en 0,7 (70\%) casos no había decalaje de tiempo, 0,1 (10\%) transcurría 1 mes, 0,05 (5\%) transcurrían de 1-3 meses, en 0,05 (5\%) transcurrían de 3-6 meses y en $0,1(10 \%)$ transcurrían más de 6 meses.

Para finalizar describiremos el tipo de tratamiento que reciben las cataratas de nuestro grupo de estudio. En el grupo de las congénitas el 58\% reciben tratamiento quirúrgico. En el grupo de las adquiridas el $90 \%$ reciben tratamiento quirúrgico (tabla III).

En las congénitas $0,56(56 \%)$ casos requirieron cirugía bilateral mientras que en 0,44 (44\%) la cirugía fue unilateral.

Se estudió el período de tiempo que transcurre entre el diagnóstico y la cirugía de las cataratas de nuestro medio.

En el grupo de las cataratas congénitas en 0,5 $(50 \%)$ casos este periodo de tiempo es menor a 1 mes, en 0,26 (26\%) es entre 1-2 meses. En el grupo

Tabla III. Tratamiento de las cataratas infantiles según su diagnóstico etiológico: cataratas congénitas y adquiridas

\begin{tabular}{llcc}
\hline Tratamiento & & \multicolumn{2}{c}{ Grupos etiologicos } \\
& & Congénitas & Adquiridas \\
\hline Quirurgico & Número & 34 & 18 \\
& Porcentaje & $58 \%$ & $90 \%$ \\
Conservador & Número & 24 & 2 \\
& Porcentaje & $42 \%$ & $10 \%$ \\
Total & Número & 58 & 20 \\
& Porcentaje & $100 \%$ & $100 \%$ \\
\hline \hline
\end{tabular}

de las adquiridas en 0,95 (95\%) casos el periodo de tiempo es menor a 1 mes (tabla IV).

\section{DISCUSIÓN}

Las cataratas pediátricas son una causa importante de pérdida de AV en la población infantil, sobre todo las congénitas y las que se desarrollan en las primeras etapas de la vida. Constituyen la primera causa de ceguera prevenible en el mundo dentro de la población pediátrica (10).

El objetivo de este estudio es conocer con más detalle las cataratas pediátricas de nuestra población con el fin de mejorar en lo posible la precocidad del diagnóstico y el tratamiento. El propósito más importante es disminuir el riesgo de desarrollo

Tabla IV. Período de tiempo que transcurre entre el diagnóstico de las cataratas infantiles quirúrgicas y el procedimiento quirúrgico

\begin{tabular}{llcc}
\hline \multirow{2}{*}{ Intervalo de tiempo } & \multicolumn{2}{c}{ Grupos etiologicos } \\
& & Congénitas & Adquiridas \\
\hline Menos de 1 mes & Número & 17 & 17 \\
\multirow{4}{*}{$1-2$ meses } & Porcentaje & $50 \%$ & $94,5 \%$ \\
& Número & 9 & 1 \\
2 2-6 meses & Porcentaje & $26,5 \%$ & $5,5 \%$ \\
\multirow{2}{*}{ Más de 6 meses } & Número & 0 & 0 \\
\multirow{2}{*}{ Total } & Porcentaje & $0 \%$ & $0 \%$ \\
& Número & 8 & 0 \\
& Porcentaje & $23,5 \%$ & $0 \%$ \\
& Número & 34 & 18 \\
\hline \hline
\end{tabular}


de una ambliopía secundaria que va a influir de forma directa en el pronóstico visual de estos niños.

Se ha realizado un estudio epidemiológico, diagnóstico y de actitud terapéutica de las cataratas infantiles de nuestro medio encontrando resultados similares a los estudios realizados en otras poblaciones $(8,9,11,12)$.

Con respecto a la etiología, en el grupo de las congénitas las más frecuentes son las aisladas (68\%). El 17\% son hereditarias, si bien es cierto que este porcentaje tendría que ser mayor, debido a que algunas de las catalogadas como aisladas podrían ser primeras mutaciones de cataratas hereditarias.

El síndrome más frecuentemente asociado es el síndrome de Down y parece que hay un incremento de la incidencia de cataratas congénitas en estos niños (2-6\%) (13). En nuestra serie los dos casos de infecciones intrauterinas asociados a catarata son rubéola congénita.

En el grupo de las adquiridas, la causa más frecuente es la traumática (traumatismos perforantes). Existen dos casos de cataratas adquiridas secundarias al tratamiento con corticoides que a su vez son los dos únicos casos de cataratas adquiridas bilaterales. La mayoría de las cataratas bilaterales son congénitas y más concretamente hereditarias.

La morfología más frecuente de las cataratas congénitas es la nuclear seguida de la polar. En las adquiridas la morfología depende de la naturaleza del traumatismo con excepción de las metabólicas que, como era de esperar, son subcapsulares posteriores. Un $27 \%$ de las cataratas congénitas presentaban otras anomalías oculares asociadas. La más frecuente era el microftalmos seguido de la persistencia de membrana pupilar. Es muy importante tener en cuenta el microftalmos como anomalía asociada más frecuente, ya que puede dificultar el tratamiento quirúrgico y la rehabilitación visual posterior. Se considera un factor de mal pronóstico visual de estos pacientes (10).

El signo clínico más frecuente de presentación en las cataratas congénitas es la leucocoria seguida del estrabismo. Estas manifestaciones clínicas son de vital importancia debido a que cada vez más, los pediatras las reconocen gracias a la incorporación de exploraciones oftalmológicas básicas en su práctica rutinaria que permiten un diagnóstico precoz.

En cuanto a la edad de presentación podemos hablar de dos picos de edad que corresponden con cada uno de los grupos de cataratas. El pico de edad de presentación de las congénitas es hacia los 3 meses mientras que en el caso de las adquiridas de nuestra serie está alrededor de los 8 años de edad.

Algunos de los factores más importantes en el pronóstico visual de estos pacientes son el diagnóstico y el tratamiento precoz de esta patología sobretodo en los niños más pequeños (14). Entendemos como precoz aquellas cataratas diagnosticadas antes de las 4-6 semanas de su aparición. En nuestra serie hemos visto que la mayoría de las cataratas congénitas así como las adquiridas tardan menos de un mes en diagnosticarse y las que son quirúrgicas, tardan menos de un mes en operarse. Estos son datos alentadores y nos indican que las técnicas de exploración para la detección precoz llevadas a cabo en los centros de atención primaria y especializada de nuestro medio están funcionando.

Sin embargo, no debemos cesar en nuestros esfuerzos por intentar que todos los niños sean diagnosticados y tratados de forma precoz, para lo cual se requiere la colaboración de padres, pediatras y oftalmólogos.

\section{BIBLIOGRAFÍA}

1. Fonseca A. Segmento anterior. In: Fonseca A, Abelairas J, Rodríguez JM, Peralta J. Actualización en cirugía oftálmica pediátrica. Madrid: Tecnimedia editorial S.L; 2000. 90-102.

2. Güell JL, Gil-Gibernau J. Catarata congénita e infantil. In: Corcóstegui B. Cirugía vitreorretiniana. Indicaciones y técnicas. Barcelona: Tecnimedia editorial S.L; 1999; 75-89.

3. Prevention of chilhood blindness. World Health Organization; 1992.

4. Foster A, Gilbert C. Epidemiology of visual impairment in childhood. In: Taylor D, ed. Practical pediatric ophthalmology. Oxford: 1997.

5. Gilbert C, Rahi J, Eckstein M, Foster A. Hereditary disease as a cause of childhood blindness: regional variation. Results of blind school studies undertaken in countries of Latin America, Asia and Africa. Ophthalmic Genet 1995; 16: 1-10.

6. Cionni RJ, Snyder ME, Osher RH. Cataract Surgery. In: Tasman W, Jaeger EA. Duane's Ophthalmology on CDROM. Philadelphia: Lippincott Williams \& Wilkins; 2001; 6: 303-563.

7. Wilson ME, Jr, Trivedi RH, Hoxie JP, Bartholomew LR. Treatment outcomes of congenital monocular cataracts: the effects of surgical timing and patching compliance. $J$ Pediatr Ophthalmol Strabismus 2003; 40: 323-329.

8. Rahi JS, Dezateux C; British Congenital Cataract Interest Group. Measuring and interpreting the incidence of congenital ocular anomalies: lessons from a national study of congenital cataract in the UK. Invest Ophthalmol Vis Sci 2001; 42: 1444-1448. 
9. Wirth MG, Russell-Eggitt IM, Craig JE, Eldel JE, Mackey DA. Aetiology of congenital and paediatric cataract in an Australian population. Br J Ophthalmol 2002; 86: 782-786.

10. Wilson ME, Pandey SK, Thakur J. Paediatric cataract blindness in the developing world: surgical techniques and intraocular lenses in the new millennium. Br J Ophthalmol 2003; 87: 14-19.

11. Amaya L, Taylor D, Russell-Eggitt I, Nischal KK, Lengyel $D$. The morphology and natural history of childhood cataracts. Surv Ophthalmol 2003; 48: 125-144.
12. González Viejo I, Ferrer Novella C, Pueyo Subías M, Melcón Sánchez B, Cuevas R, Bada T, et al. Cataratas congénitas y adquiridas infantiles en nuestro medio. Arch Soc Esp Oftalmol 1999; 74: 627-630.

13. Cassidy L, Taylor D. Congenital cataract and multisystem disorders. Eye 1999; 13: 464-473.

14. Taylor D, Wright KW, Amaya L, Cassidy L, Nischal K, Russell-Eggitt I, et al. Should we aggressively treat unilateral congenital cataracts? Br J Ophthalmol 2001; 85: 1120-1126. 